\title{
miR-363-3p inhibits rat lung alveolar type II cell proliferation by downregulating STRA6 expression and induces cell apoptosis via cellular oxidative stress and G1-phase cell cycle arrest
}

\author{
Jintao Zheng ${ }^{1} \wedge$, Shibo Zhu ${ }^{2}$, Huiyu Xu ${ }^{1}$, Jiequan Li ${ }^{1}$, Huajian Tang ${ }^{1}$, Yanfen Zhou ${ }^{1}$, Zhaomei Huang ${ }^{1}$, \\ Guoqing Liu ${ }^{1,3}$
}

\begin{abstract}
${ }^{1}$ Department of Neonatal and Pediatric Surgery, Foshan Women and Children Hospital Affiliated to Southern Medical University, Foshan, China; ${ }^{2}$ Department of Pediatric Surgery, Guangzhou Women and Children's Medical Center, Guangzhou Medical University, Guangzhou, China; ${ }^{3}$ Women and Children Medical Research Center Affiliated to Foshan Institute of Fetal Medicine, Foshan, China

Contributions: (I) Conception and design: J Zheng, G Liu; (II) Administrative support: G Liu; (III) Provision of study materials or patients: J Zheng, S Zhu; (IV) Collection and assembly of data: H Xu, J Li, Y Zhou, Z Huang; (V) Data analysis and interpretation: J Zheng, H Tang; (VI) Manuscript writing: All authors; (VII) Final approval of manuscript: All authors.

Correspondence to: Guoqing Liu. Department of Neonatal and Pediatric Surgery, Foshan Women and Children Hospital Affiliated to Southern Medical University, No. 11 West Renmin Rd, Chancheng District, Foshan 528000, China. Email: liuguoqing-fs@hotmail.com; Jintao Zheng. Department of Neonatal and Pediatric Surgery, Foshan Women and Children Hospital Affiliated to Southern Medical University, No. 11 West Renmin Rd, Chancheng District, Foshan 528000, China. Email: zheng-jintao@foxmail.com.
\end{abstract}

Background: miR-363-3p, the retinoid signaling pathway (RSP), and its associated membrane receptor, stimulated by retinoic acid 6 (STRA6), participate in lung development. We hypothesize that miR-363-3p is involved in lung cell proliferation and apoptosis by regulating the expression of STRA6, and this study was designed to investigate the effect of changes in the expressions of miR-363-3p and the STRA6 gene on the proliferation and apoptosis of rat alveolar type II cells.

Methods: To confirm our hypothesis, we used: a dual-luciferase reporter assay; cell culture and transfection; real-time quantitative polymerase chain reaction (PCR); Western blotting; a cell proliferation assay and flow cytometry analysis of the cell cycle, cell apoptosis, oxidative stress level, and mitochondrial membrane potential.

Results: Our results showed that STRA6 is a target gene for miR-363-3p, and when the expression of miR363-3p increased, the relative messenger RNA (mRNA) expression of STRA6 decreased, which caused a decrease in STRA6 protein synthesis and subsequent inhibition of rat lung alveolar type II cell proliferation. In contrast, inhibiting the expression of miR-363-3p promoted the proliferation of these cells. This study also found that an increased expression of miR-363-3p induced rat lung alveolar type II cell apoptosis led to an increase in the oxidative stress level, decreased mitochondrial membrane potential, and an inducement of G1-phase cell cycle arrest.

Conclusions: In conclusion, miR-363-3p is associated with lung cell proliferation and apoptosis, while miR-363-3p inhibits rat lung alveolar type II cell proliferation by downregulating the expression of STRA6 and induces cell apoptosis by increasing cellular oxidative stress and G1-phase cell cycle arrest.

Keywords: miR-363-3p; stimulated by retinoic acid 6 (STRA6); retinoid signaling pathway (RSP); lung development

Submitted Jun 21, 2021. Accepted for publication Jul 22, 2021.

doi: $10.21037 /$ tp-21-303

View this article at: https://dx.doi.org/10.21037/tp-21-303

^ ORCID: 0000-0003-0667-0067. 


\section{Introduction}

The retinoid signaling pathway (RSP) is one of the most significant signaling pathways in lung development. Retinol [vitamin A (VA)] and its active metabolite, retinoic acid (RA), regulate the proliferation, differentiation, patterning, and maturation of lung cells by regulating the expression of target genes, thereby affecting lung specification, branching morphogenesis, and alveolarization (1). Retinol metabolism abnormalities may lead to a variety of congenital lung malformations, such as alveolar capillary hypoplasia, pulmonary agenesis, lung hypoplasia, and congenital diaphragmatic hernia $(\mathrm{CDH})$ with lung hypoplasia, regardless of whether an embryo is deficient in or has excess retinol $(2,3)$. Stimulated by retinoic acid $6(S T R A 6)$ is a specific retinol-binding protein ( $\mathrm{RBP})$ receptor of retinol in a cell membrane, and it is a key factor in regulating the transport and uptake activity of retinol in the RSP (4). Our previous research showed that in a rat model of nitrofen-induced $\mathrm{CDH}$ with lung hypoplasia, downregulating STRA6 expression reduces rat lung alveolar type II cell proliferation (5) and affects retinol absorption via the RSP (6). In addition, Chen et al. (7) found that STRA6 expression is associated with apoptosis.

MicroRNA (miRNA) has been identified as playing a crucial role in lung development, and it regulates the expression of target genes at the post-transcriptional level by targeting its messenger RNA (mRNA) (8). For example, Lu et al. (9) found that Dev transgenic overexpression of the miRNA miR-17-92 cluster promotes lung epithelial progenitor cells proliferation and inhibits its differentiation. Ventura et al. (10) discovered that deletion of miR-17-92 cluster in mice cause immediate postnatal death with smaller embryos and hypoplastic lungs. Another of our previous studies showed that miR-363 expression is increased in the fetal lungs of a rat model with nitrofen-induced $\mathrm{CDH}$ with lung hypoplasia, this being determined by a miRNA microarray analysis using an Agilent miRNA array (11). miRNA software predicted that miR-363-3p is the upstream miRNA of the STRA6 gene. Therefore, we hypothesize that miR-363-3p is involved in lung cell proliferation and cell apoptosis by regulating STRA6 expression. Therefore, this study aims to determine the relationship between miR-363$3 \mathrm{p}$ and the STRA6 gene of the RSP in lung cell proliferation and apoptosis.

We present the following article in accordance with the MDAR reporting checklist (available at https://dx.doi. org/10.21037/tp-21-303).

\section{Methods}

\section{Dual luciferase reporter assay}

Four micrograms of dual luciferase vector (STRA6-wt or STRA6-mut) and $100 \mathrm{nmol} / \mathrm{L}$ of $\mathrm{miR}-363-3 \mathrm{p}$ mimic [or mimic negative control (NC), miR-363-3p inhibitor, or inhibitor NC] were co-transfected into human embryonic kidney 293T (HEK293T) cells. Luciferase activity was measured by using a dual luciferase reporter gene assay kit (Beyotime, Shanghai, China) after 48 hours of transfection. Normalized firefly luciferase activity was compared among the groups. STRA6-wt and STRA6-mut [containing the STRA6 3' untranslated region (UTR) fragment] were synthesized by Guangzhou all-Perfect Biological Technology Co., Ltd., China. Transfections were repeated independently at least 3 times.

\section{Cell culture and transfection}

Rat lung alveolar type II cells (RLE-6TN, ATCC ${ }^{\circledR}$ number: CRL-2300 ${ }^{\mathrm{TM}}$, USA) were cultured in complete Roswell Park Memorial Institute (RPMI) 1640 medium [supplemented with $100 \mathrm{U} / \mathrm{mL}$ of penicillin, $100 \mu \mathrm{g} / \mathrm{mL}$ of streptomycin, and $10 \%$ fetal bovine serum (FBS)]. When the cells reached $90 \%$ confluence, they were digested with $0.25 \%$ trypsin. After the digestion process was completed, add the medium to stop the digestion. The cells were centrifuged at $500 \times \mathrm{g}$ for 2 minutes and the supernatant was discarded. The cells were then collected, resuspended in medium, and spread on a cell culture plate or cell culture flask for additional culturing and passaging.

Rat lung alveolar type II cells were divided into a control group, NC group, miR-363-3p mimic group, and miR363-3p inhibitor group. Rat lung alveolar type II cells from each group were cultured in 6-well plates overnight and then transfected with either $100 \mathrm{nM}$ of control, NC, miR363-3p mimic, or miR-363-3p inhibitor (Guangzhou AllPerfect Biological Technology, China) with Lipofectamine 3000 (Invitrogen, USA). The successfully transfected rat lung alveolar type II cells were used in various subsequent experiments at different time points.

\section{Total RNA extraction, Reverse Transcription-Polymerase Chain Reaction and qPCR}

Total RNA was isolated from the cells using a Total RNA Miniprep Kit and then reverse transcribed into complementary DNA (cDNA) using a MightyScript First Strand cDNA 
Table 1 Sequences of the primers

\begin{tabular}{ll}
\hline Name of primer & Primer sequence \\
\hline STRA6-forward & 5'-GAGGACTCACAGGGCCAATC-3' \\
STRA6-reverse & 5'-GACTATGGCTTGGCGAGAGG-3' \\
$\beta$-Actin-forward & 5'-AGGGTGTGATGGTGGGTATGG-3' \\
$\beta$-Actin-reverse & 5'-AGTTGGTGACAATGCCGTGTTC-3' \\
miR-363-3p & 5'-AATTGCACGGTATCCATCTGT-3' \\
U6 & 5'-GTCGTCACACAACTGGGCTT-3'
\end{tabular}

STRA6, stimulated by retinoic acid 6 .

Synthesis Master Mix (Tailing Reaction). mRNA was detected with a $2 \times$ SYBR Green Abstart polymerase chain reaction (PCR) mix, and miRNA was detected with a microRNA quantitative PCR (qPCR) Kit (SYBR Green method) using a fluorescence qPCR system (CFX96, Bio-Rad, USA). The reaction system was prepared, and the reaction conditions were set according to the instruction manual. The primer sequences are shown in Table 1. The abovementioned reagents were obtained from GeneCopoeia, USA. The experiments were all run in duplicate for each sample and primer pair.

\section{Western blotting}

Total protein was extracted with radioimmunoprecipitation assay (RIPA) buffer (Sigma-Aldrich, USA), and the protein concentration was measured using a Bicinchoninic Acid Kit for Protein Determination (Sigma-Aldrich, USA) according to the manufacturer's protocol. A $10 \%$ sodium dodecyl sulfate (SDS) separation gel and a concentration gel were prepared with an SDS-polyacrylamide (PAGE) gel quick preparation kit (Beyond, Shanghai, China). Glyceraldehyde 3 -phosphate dehydrogenase $(G A P D H)$ was used as an internal reference protein. Thirty microliters of protein sample were mixed with SDS-PAGE loading buffer, then the proteins were separated on the concentration gel by SDS-PAGE and transferred onto nitrocellulose membranes which were incubated overnight at $4{ }^{\circ} \mathrm{C}$ with a goat anti-rat STRA6 primary antibody (Affinity Biosciences, USA) and an anti-GAPDH antibody (Everest Biotech, UK).

The blots were subsequently incubated with a horseradish peroxidase-linked rabbit anti-goat immunoglobulin G $(\mathrm{IgG})(\mathrm{H}+\mathrm{L})$ secondary antibody (Thermo Fisher Scientific, USA). Peroxidase-positive bands were visualized with enhanced chemiluminescence (ECL) reagents (Bio-Rad, USA). Changes in protein expression were quantified, while images were taken using a Bio-Rad Gel Doc XR+ gel imaging system (Bio-Rad, USA). The abovementioned experimental procedures were carried out according to the manufacturer's instructions.

\section{Cell proliferation assay}

A Cell Counting Kit 8 (CCK-8) (Sigma-Aldrich, USA) was used to assess cell proliferation. Transfected rat lung alveolar type II cells from each group were transferred to a 96-well plate and cultured for 12, 24, 36, or 48 hours. Twenty microliters of CCK-8 reagent were added to each well, and the cells were then incubated in a humidified atmosphere of $5 \%$ carbon dioxide for 30 minutes at $37^{\circ} \mathrm{C}$. One hundred microliters of supernatant were aspirated from each well for analysis. The absorbance of the cell sample at a wavelength of $450 \mathrm{~nm}$ was measured with a Sunrise microplate reader (Tecan Group, Ltd., Mannedorf, Switzerland).

\section{Flow cytometry analysis of cell apoptosis}

After rat lung alveolar type II cells were successfully transfected with the control, NC, miR-363-3p mimic, or miR-363-3p inhibitor for 48 hours, the cells were digested with trypsin without ethylenediaminetetraacetic acid (EDTA). After the digestion was terminated, the cells were gently pipetted and transferred to a $1.5 \mathrm{~mL}$ EP tube and centrifuged at $1,000 \times \mathrm{g}$ for 5 minutes; the supernatant was discarded, and the cells were washed with $1 \mathrm{~mL}$ of phosphate buffer saline (PBS), rinsed gently, and centrifuged at 1,000 $\times \mathrm{g}$. The cells were rinsed 3 times for 5 minutes, the supernatant was discarded, $195 \mu \mathrm{L}$ of Annexin $\mathrm{V}$ fluorescein isothiocyanate (V-FITC) binding solution (Guangzhou All-Perfect Biological Technology, China) was added, and the cells were gently resuspended. Five microliters of Annexin V-FITC was added, and the cells were gently mixed. Then, $10 \mu \mathrm{L}$ of propidium iodide (PI) staining solution (Guangzhou All-Perfect Biological Technology, China) was added and the cells were gently mixed. The cells were incubated at room temperature $\left(20-25{ }^{\circ} \mathrm{C}\right)$ in the dark for 10-20 minutes and placed in an ice bath. A FACSCalibur flow cytometer (Agilent Technologies, Inc., USA) was used for the analysis and NovoExpress software was used to analyze the percentage of apoptotic cells.

\section{Flow cytometry analysis of the oxidative stress level}

Rat lung alveolar type II cells were collected after successful 
transfection with the control, NC, miR-363-3p mimic, or miR-363-3p inhibitor for 48 hours. 2'-7' dichlorofluorescin diacetate (DCFH-DA) (Guangzhou All-Perfect Biological Technology, China) was diluted with serum-free culture medium at a 1:1,000 ratio to a achieve final concentration of $10 \mu \mathrm{mol} / \mathrm{L}$. Next, $1 \mathrm{~mL}$ of DCFH-DA was added to stain the cells, and the cells were incubated in a cell incubator at $37{ }^{\circ} \mathrm{C}$ for 20 minutes. The cells were mixed by inversion every 5 minutes to ensure full contact between the probe and the cells. The cells were then washed 3 times with serum-free cell culture medium to remove the DCFH-DA that had not entered the cells fully. A FACSCalibur flow cytometer (Agilent Technologies, Inc. USA) was used for the analysis, and NovoExpress software was used to analyze the oxidative stress level of the cells.

\section{Flow cytometry analysis of the mitochondrial membrane potential}

After rat lung alveolar type II cells were successfully transfected with the control, NC, miR-363-3p mimic, or miR-363-3p inhibitor for 48 hours, $1 \mathrm{~mL}$ of JC- 1 dye was added for 30 minutes to stain the cells. The cells were then rinsed 3 times with a basal medium. The cells were collected and resuspended in $500 \mu \mathrm{L}$ of PBS. A FACSCalibur flow cytometer (Agilent Technologies, Inc.) was used for the analysis and NovoExpress software was used to analyze the mitochondrial membrane potential.

\section{Flow cytometry analysis of the cell cycle}

After rat lung alveolar type II cells were successfully transfected with the control, NC, miR-363-3p mimic, or miR-363-3p inhibitor, the cells were digested with trypsin and harvested for 48 hours. The cells were centrifuged at $1,000 \times \mathrm{g}$ for 3 minutes to pellet the cells. One milliliter of ice-cooled PBS was added, and the cells were resuspended and transferred to a $1.5 \mathrm{~mL}$ centrifuge tube. The cells were centrifuged again, the supernatant was carefully aspirated, and the bottom of the centrifuge tube was gently flicked to disperse the cells to avoid the cells clumping properly. Next, $500 \mu \mathrm{L}$ of $70 \%$ cold ethanol was added to the cells, and they were fixed overnight at $4{ }^{\circ} \mathrm{C}$. The fixative solution was discarded, $1 \mathrm{~mL}$ of PBS was added to resuspend the cells, the cells were centrifuged at $500 \times \mathrm{g}$ at $4^{\circ} \mathrm{C}$ for 5 minutes, the supernatant was discarded, $500 \mu \mathrm{L}$ of PI/ RNase A staining working solution (Guangzhou All-Perfect Biological Technology, China) was added, and the cells were incubated at room temperature in the dark for 60 minutes. A FACSCalibur flow cytometer (Agilent Technologies, Inc., USA) was used for the analysis, and NovoExpress software was used to analyze the cell cycle.

\section{Statistical analysis}

Statistical analyses were performed using SPSS Statistics (version 12.0.0.0). All numerical data were presented as the mean \pm SD. Differences between two groups were tested using an unpaired $t$-test. Statistical significance was defined as a $\mathrm{P}$ value of less than 0.05 .

\section{Results}

\section{$m i R-363-3 p$ regulates $S T R A 6$ expression}

Analysis of the expression of the dual luciferase reporter gene showed that reporter gene fluorescence was significantly decreased (by 19\%) in the STRA6-wt + miR363-3p group compared with the STRA6-wt mimic NC group $(\mathrm{P}=0.04)$. In contrast, reporter gene fluorescence was significantly increased (by $32 \%$ ) in the STRA6-wt + miR363-3p inhibitor group compared with the STRA6-wt + inhibitor NC group $(\mathrm{P}=0.013)$. This result indicates that a change in miR-363-3p expression can alter the fluorescence of a dual luciferase vector containing the STRA6 3'UTR fragment that binds to the target STRA6 (Figure 1).

\section{miR-363-3p inhibits rat lung alveolar type II cell proliferation by downregulating STRA6 expression}

The qPCR and Western blot results showed no statistically significant differences in the relative expression of miR363-3p, STRA6 mRNA or STRA6 protein between the control and NC groups [miR-363-3p: 1.017 \pm 0.224 "versus" $0.942 \pm 0.117, \mathrm{P}=0.633$ (>0.05); STRA6 mRNA: $1.001 \pm 0.055$ "versus" $0.960 \pm 0.029, \mathrm{P}=0.316(>0.05)$; STRA6 protein: $86.267 \pm 2.311$ "versus" 82.323 $\pm 2.751, \mathrm{P}=0.247(>0.05)$ ].

Compared with the control group, the relative expression of $\mathrm{miR}-363-3 \mathrm{p}$ in the $\mathrm{miR}-363-3 \mathrm{p}$ mimic group $(2.053 \pm 0.397)$ was significantly increased $[\mathrm{P}=0.017$ $(<0.05)]$, and the relative expressions of STRA6 mRNA and STRA6 protein in the miR-363-3p mimic group (STRA6 mRNA: $0.215 \pm 0.007$; STRA6 protein: $48.430 \pm 4.060)$ was significantly decreased $[\mathrm{P}=0.000$ and $\mathrm{P}=0.001(<0.05)$, respectively].

Compared with the control group, the relative expression of 


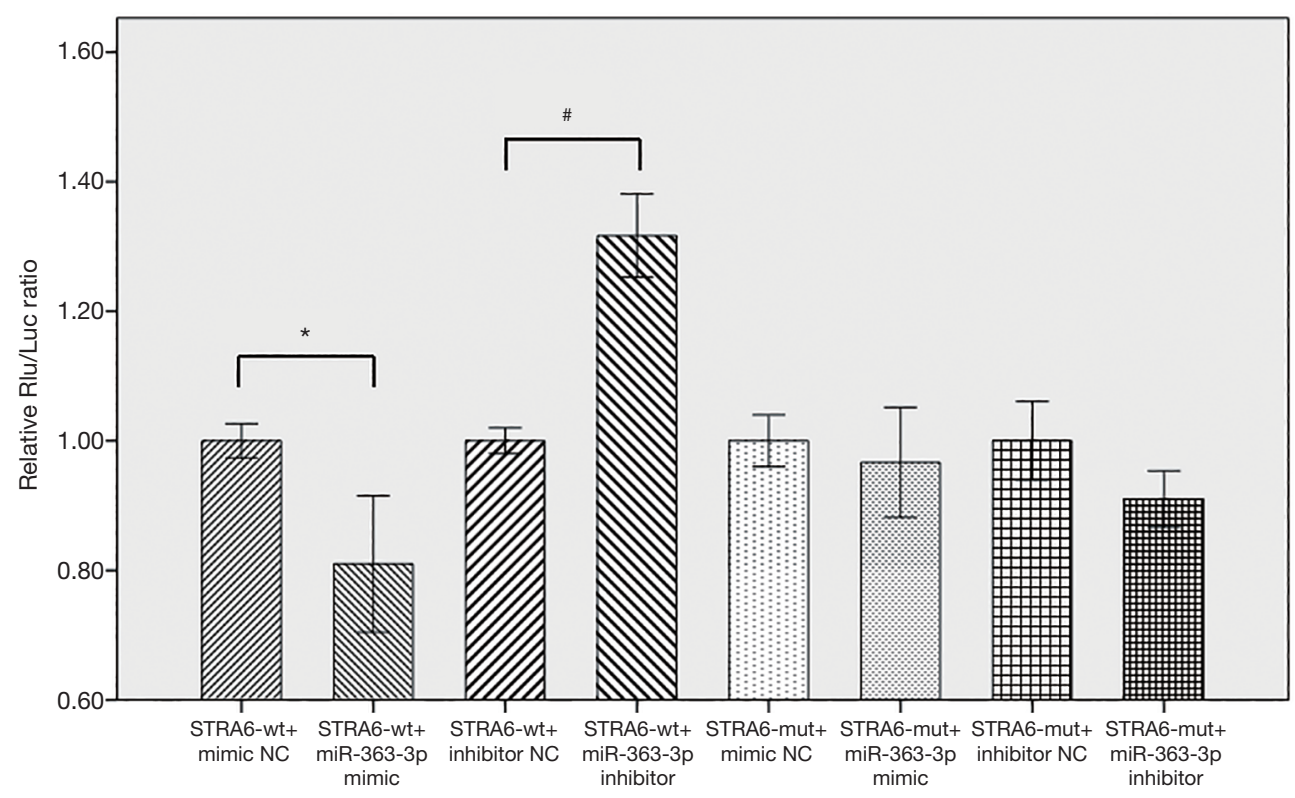

Figure 1 Reporter gene fluorescence was significantly decreased (by 19\%) in the STRA6-wt + miR-363-3p group compared with the STRA6-wt mimic NC group (P=0.04). In contrast, reporter gene fluorescence was significantly increased (by $32 \%)$ in the STRA6-wt + miR363-3p inhibitor group compared with the STRA6-wt inhibitor NC group ( $\mathrm{P}=0.013)$. * and * indicate $\mathrm{P}<0.05$ between the STRA6-wt + mimic NC and STRA6-wt + miR-363-3p mimic groups and between the STRA6-wt + inhibitor NC and STRA6-wt + miR-363-3p inhibitor groups, respectively. STRA6, stimulated by retinoic acid 6; NC, negative control.

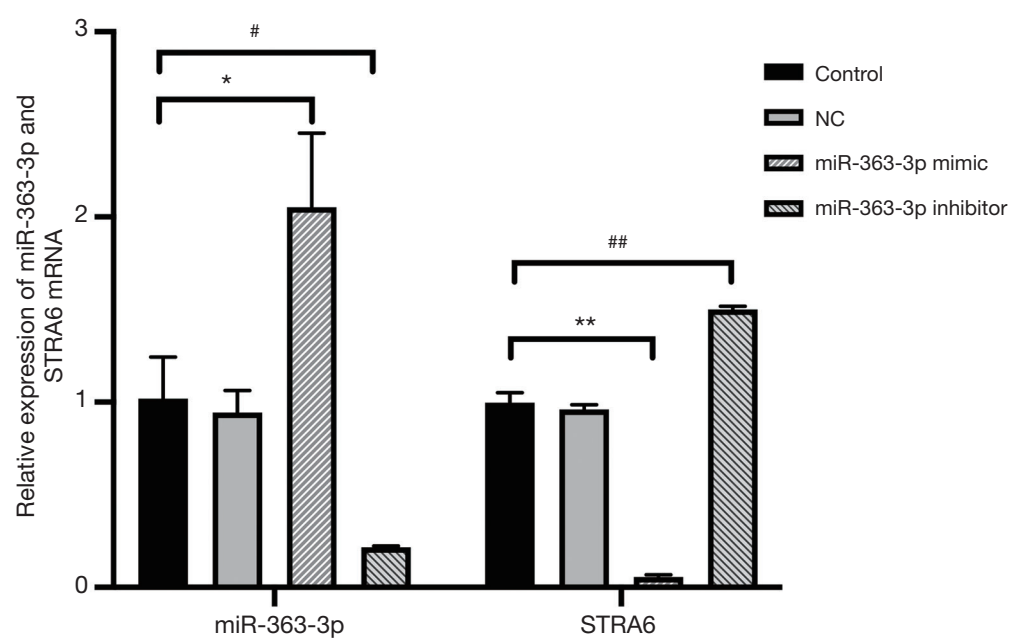

Figure 2 Compared with in the control group, the relative expression of miR-363-3p in the miR-363-3p mimic group was significantly higher and the relative expression of STRA6 mRNA in the miR-363-3p mimic group was significantly decreased. The relative expression of miR-363-3p was significantly lower and the relative mRNA expression of STRA6 was significantly higher in the miR-363-3p inhibitor group than in the control group. *, ", ** and ${ }^{\# \#}$ indicate $\mathrm{P}<0.05$. STRA6, stimulated by retinoic acid 6.

miR-363-3p in the miR-363-3p inhibitor group $(0.057 \pm 0.009)$ was significantly decreased $[\mathrm{P}=0.002(<0.05)]$ and the relative expressions of STRA6 mRNA and STRA6 protein in the miR- 363-3p inhibitor group (STRA6 mRNA: $1.498 \pm 0.018$ and STRA6 protein: $110.283 \pm 7.889)$ was significantly increased $[\mathrm{P}=0.000$ and $\mathrm{P}=0.025(<0.05)$, respectively] (Figures 2 and 3). 
A

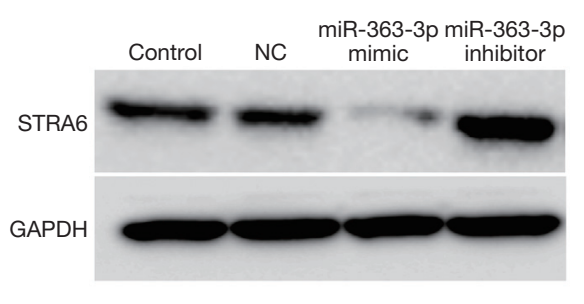

B

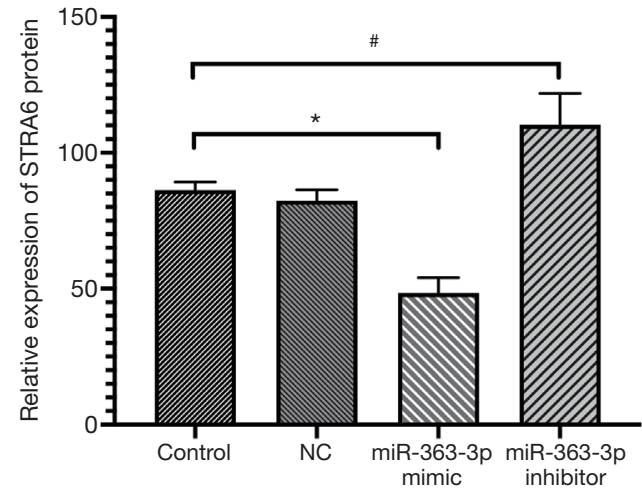

Figure 3 Compared with in the control group, the relative protein expression of STRA6 in the miR-363-3p mimic group was significantly decreased, and in the miR-363-3p inhibitor group was significantly increased. * and ${ }^{\#}$ indicate $\mathrm{P}<0.05$. STRA6, stimulated by retinoic acid 6.

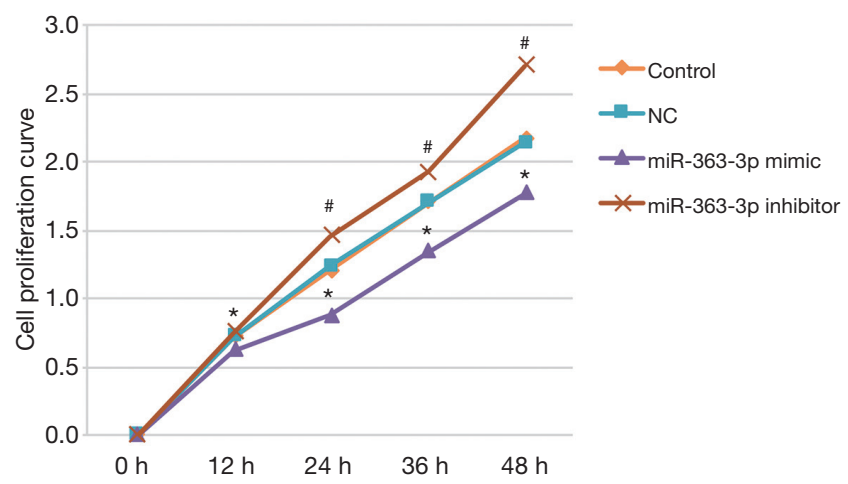

Figure 4 Rat lung alveolar type II cell proliferation was significantly decreased in the miR-363-3p mimic group compared with the control group at 12, 24, 36 and 48 hours post-transfection and was significantly increased in the miR-363-3p inhibitor group compared with the control group at 24, 36 and 48 hours posttransfection. ${ }^{*}$ and ${ }^{\#}$ indicate $\mathrm{P}<0.05$.

The cell proliferation assay results demonstrated no statistically significant difference in rat lung alveolar type II cell proliferation between the control and NC groups at 12, 24,36 , or 48 hours post-transfection (12 hours: $0.720 \pm 0.034$ "versus" $0.721 \pm 0.033, \mathrm{P}=0.982 ; 24$ hours: $1.202 \pm 0.046$ "versus" $1.238 \pm 0.081, \mathrm{P}=0.513 ; 36$ hours: $1.705 \pm 0.034$ "versus" $1.703 \pm 0.040, \mathrm{P}=0.959$; 48 hours: $2.174 \pm 0.066$ "versus" 2.138 $\pm 0.078, \mathrm{P}=0.535)$.

Compared with the control group, rat lung alveolar type II cell proliferation was significantly decreased in the miR-363-3p mimic group at 12, 24, 36, and 48 hours post-transfection (12 hours: control "versus" $0.623 \pm 0.023$, $\mathrm{P}=0.003 ; 24$ hours: control "versus" $0.875 \pm 0.057$, $\mathrm{P}=0.000 ; 36$ hours: control "versus" $1.342 \pm 0.069$,
$\mathrm{P}=0.000 ; 48$ hours: control "versus" $1.772 \pm 0.037$, $\mathrm{P}=0.000$.). Rat lung alveolar type II cell proliferation was significantly increased in the miR-363-3p inhibitor group compared with the control group at 24,36 , and 48 hours post-transfection (24 hours: control "versus" $1.460 \pm 0.065$, $\mathrm{P}=0.001 ; 36$ hours: control "versus" $1.928 \pm 0.025$, $\mathrm{P}=0.000 ; 48$ hours: control "versus" $2.700 \pm 0.064$, $\mathrm{P}=0.000$ ) (Figure 4).

\section{miR-363-3p induces cell apoptosis by increasing cellular oxidative stress and G1-phase cell cycle arrest}

Flow cytometry analysis of cell apoptosis demonstrated that the percentages of apoptotic cells (Q2-2 + Q2-4) in the control group, NC group, miR-363-3p mimic group, and miR-363-3p inhibitor group were $8.93 \%, 9.10 \%$, $21.49 \%$, and $4.64 \%$, respectively. The percentage of apoptotic cells in the miR-363-3p mimic group increased by $12.56 \%$ compared with that of the control group (21.49\% versus $8.93 \%)$. The percentage of apoptotic cells in the miR-363-3p inhibitor group was decreased by $4.29 \%$ compared with that of the control group $(8.93 \%$ "versus" 4.64\%) (Figure 5).

Flow cytometry analysis of the oxidative stress level results showed that the oxidative stress levels in the control group, NC group, miR-363-3p mimic group, and miR$363-3 p$ inhibitor group were $27.03 \%, 29.53 \%, 39.53 \%$, and $19.45 \%$, respectively. The oxidative stress level in the miR$363-3 \mathrm{p}$ mimic group was increased by $12.50 \%$ compared with that of the control group (39.53\% "versus" $27.03 \%)$. The oxidative stress level in the miR-363-3p inhibitor group was decreased by $7.58 \%$ compared with that of the control group (27.03\% "versus" 19.45\%) (Figure 6). 

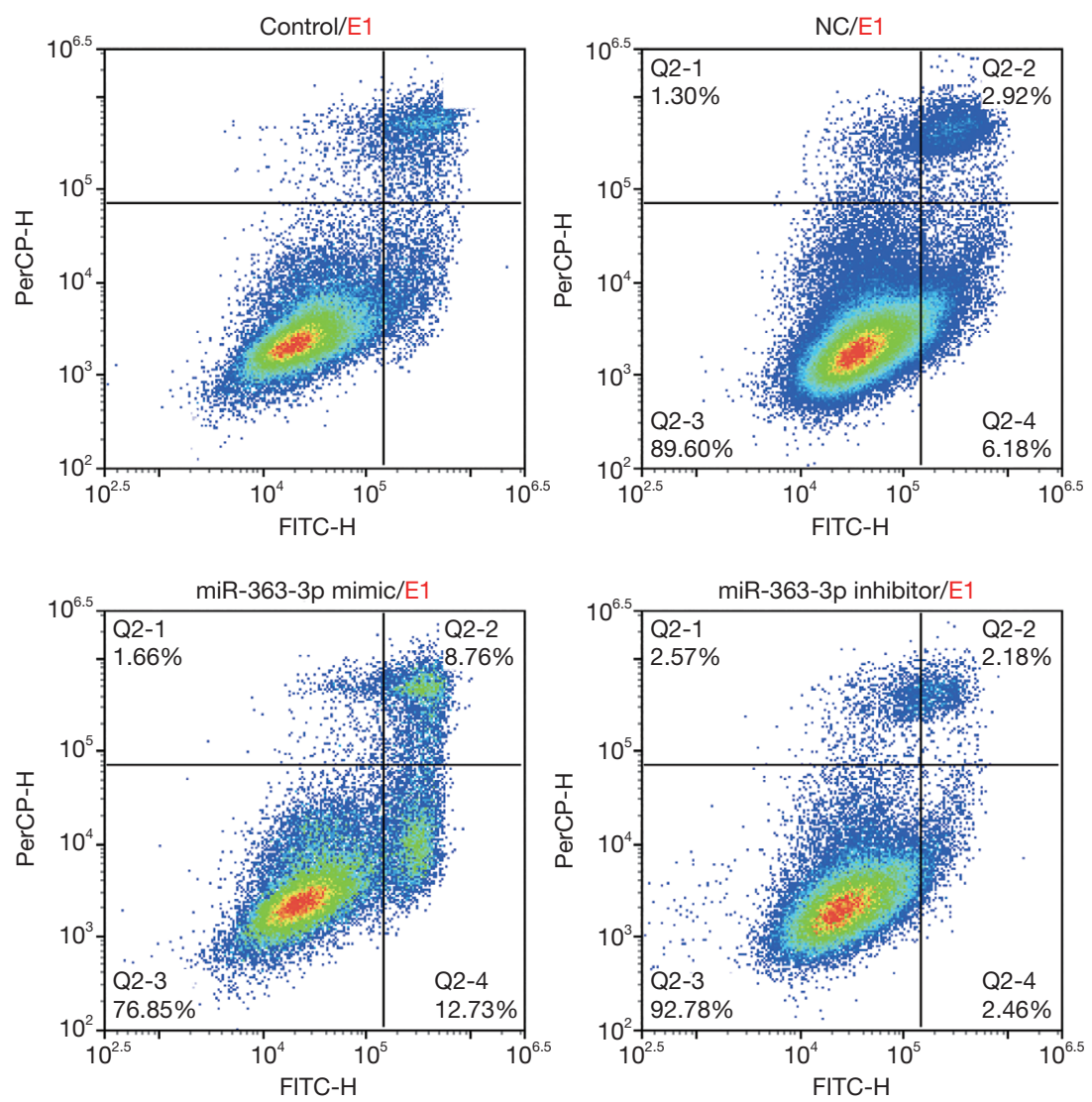

Figure 5 The percentage of cell apoptosis in the miR-363-3p mimic group increased by $12.56 \%$ compared with in the control group (21.49\% "versus" $8.93 \%)$. The percentage of apoptotic cells in the miR-363-3p inhibitor group was decreased by $4.29 \%$ compared with that of the control group (8.93\% "versus" $4.64 \%)$.

Flow cytometry analysis of the mitochondrial membrane potential proved that in the miR-363-3p mimic group, it was decreased by $10.20 \%$ compared with that of the control group (90.56\% "versus" 80.36\%) (Figure 7).

Flow cytometry analysis of the cell cycle showed that compared with that of the control group, the Freq G1 value in the miR-363-3p mimic group was increased by 3.93 (43.42 "versus" 39.49), and the Freq G1 value in the miR-363-3p inhibitor group was decreased by 6.09 (39.49 "versus" 33.40). Therefore, miR-363-3p was shown to induce rat type II alveolar cell apoptosis by eliciting G1phase cell cycle arrest (Figure 8).

\section{Discussion}

miRNA, the RSP, and the associated RBP membrane receptor STRA6 are involved in lung development. It is known that miRNA plays a crucial role in the cell cycle, cell proliferation, and cell apoptosis, including lung development, by regulating the expression of complementary mRNA $(8,12)$. During embryogenesis, the RSP is involved in the development of the trachea, airway, lungs and diaphragm. RA is a biologically active metabolite of VA and is considered a key regulator in the respiratory system development (13). STRA6 is a specific RBP membrane receptor of VA and it is a major physiological mediator of cellular VA uptake in the RSP (14). Mutations in the STRA6 gene can cause congenital lung malformations, including alveolar capillary hypoplasia, lung hypoplasia, and CDH with lung hypoplasia (15).

Our previous research demonstrated that increased miR-363 expression is associated with lung hypoplasia in a nitrofen-treated rat model (11). miRNA software (http://www.targetscan.org) predicted that miR-363 is a regulatory factor of the $S T R A 6$ gene. In this study, we proved that miR-363-3p regulates STRA6 expression using 

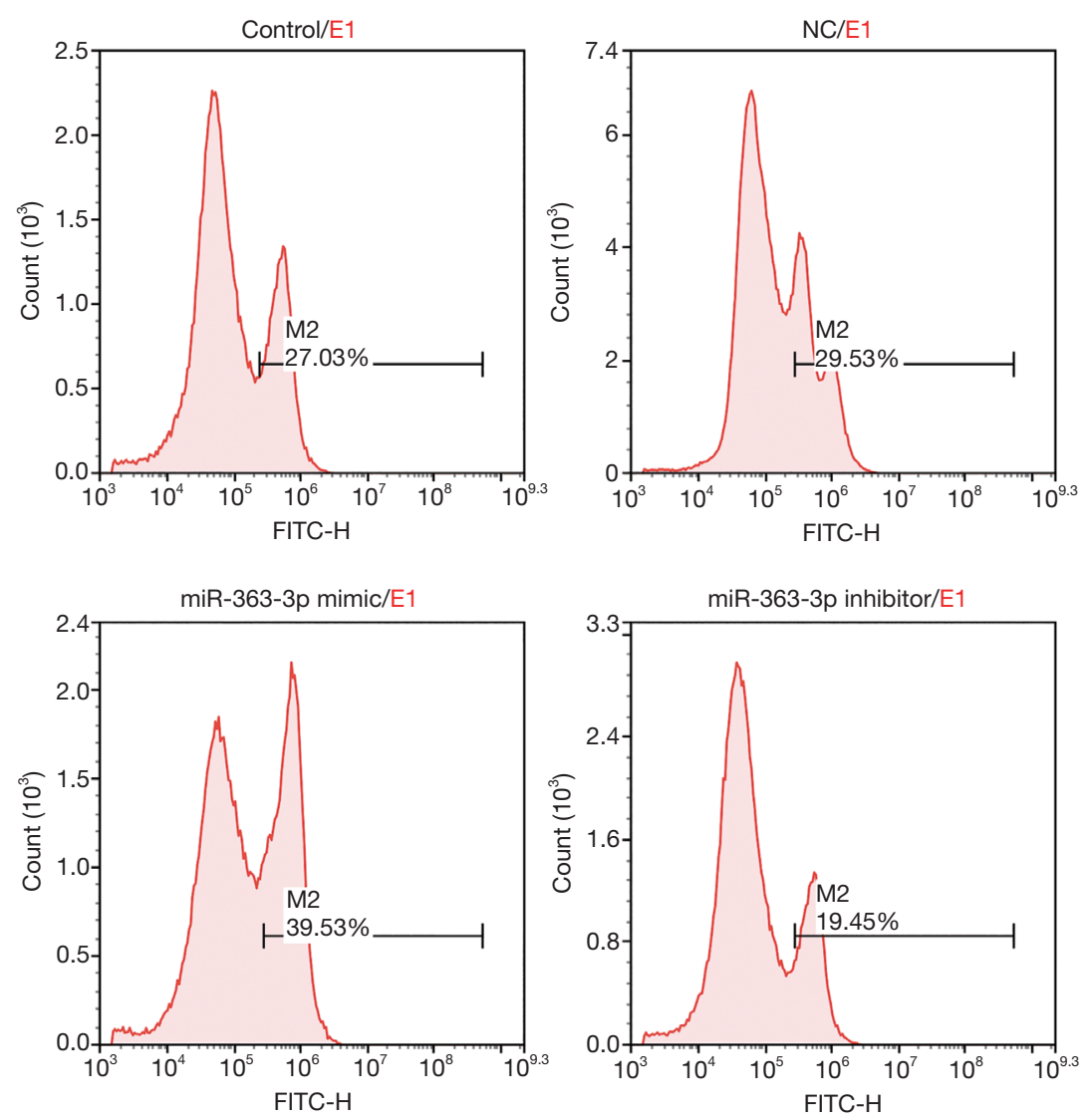

Figure 6 The oxidative stress level in the miR-363-3p mimic group was increased by $12.50 \%$ compared with in the control group (39.53\% "versus" $27.03 \%$ ). The oxidative stress level in the miR-363-3p inhibitor group was decreased by $12.50 \%$ compared with in the control group (27.03\% "versus" $7.58 \%)$.

a dual luciferase reporter assay. Our previous research showed that inhibiting STRA6 expression leads to reduced rat lung alveolar type II cell proliferation (5). Therefore, in this study we first overexpressed or inhibited miR-363$3 \mathrm{p}$ in rat alveolar type II cells by transfecting the cells with either a miR-363-3p mimic or miR-363-3p inhibitor. Then, STRA6 mRNA levels, STRA6 protein levels, and cell proliferation were measured by qPCR, Western blotting, and a cell proliferation assay, respectively. Consistent with the regulatory mechanisms of miR$363-3 p$, the results showed that when miR-363-3p was overexpressed in rat alveolar type II cells from the miR363-3p mimic group, the relative expression of STRA6 mRNA was decreased, leading to a decrease in STRA6 protein synthesis and vice versa.

Moreover, the proliferation of rat alveolar type II cells in the miR-363-3p mimic group was significantly decreased. When the expression of miR-363-3p was inhibited in the miR-363-3p inhibitor group, cell proliferation was significantly enhanced. Therefore, we hypothesized that miR-363-3p inhibits rat lung alveolar type II cell proliferation by downregulating STRA6 expression based on the above experiments.

Oxidative stress is a mechanism of cell apoptosis, and its increase can induce cell apoptosis. Mitochondrial membrane potential can reflect oxidative stress levels, and as the oxidative stress level increases, mitochondrial membrane potential decreases (16). This study found that the percentage of apoptotic rat alveolar type II cells was positively correlated with the expression level of miR-363$3 \mathrm{p}$, and the percentage of these cells increased significantly when miR-363-3p was overexpressed. Furthermore, analysis of oxidative stress levels by flow cytometry showed that when the percentage of apoptotic cells increased in the miR-363-3p mimic group, the DCFH-DA fluorescent signal was stronger, which indicated that the oxidative 

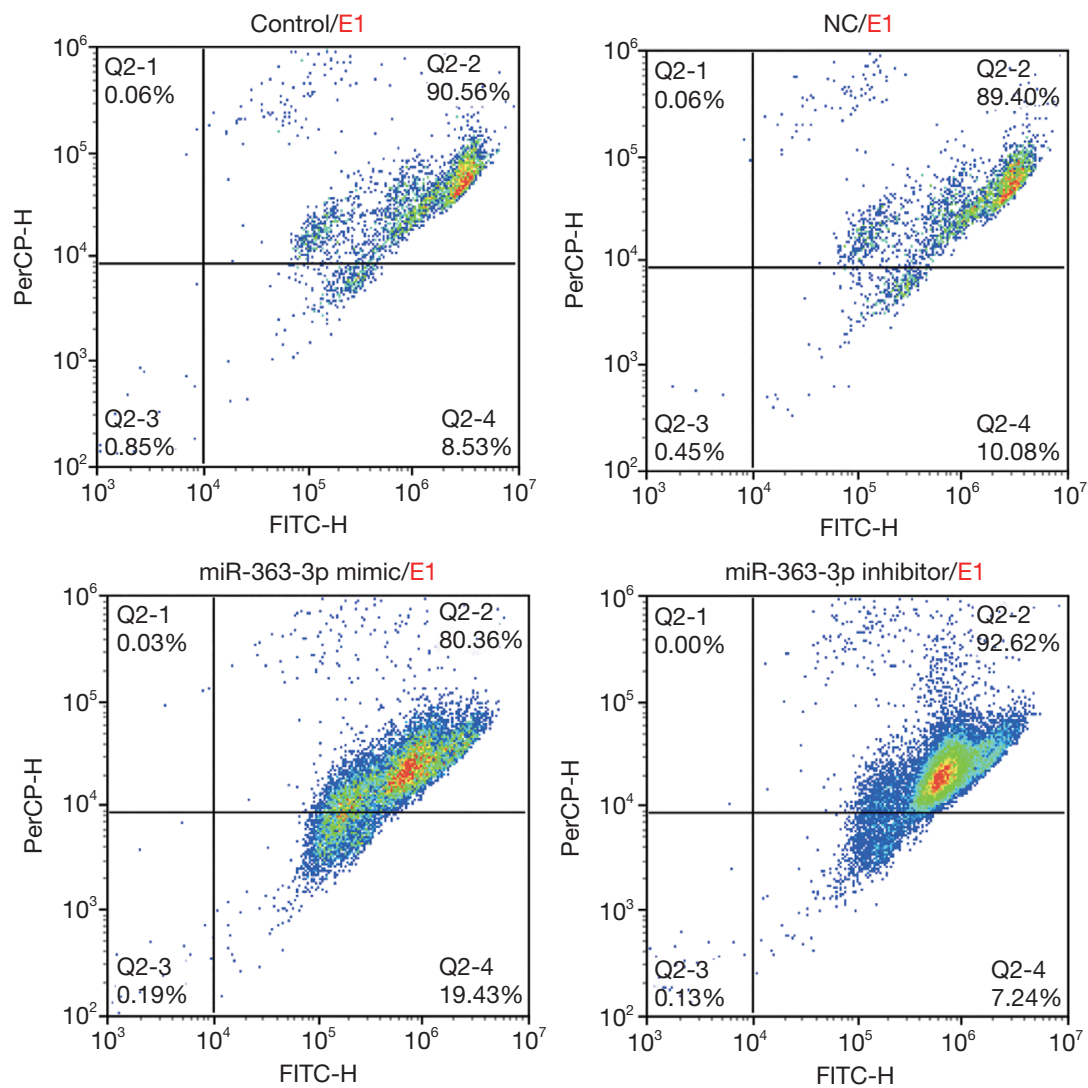

Figure 7 Mitochondrial membrane potential in the miR-363-3p mimic group was decreased by $10.20 \%$ compared with in the control group (90.56\% “versus" $80.36 \%)$.

stress level in this group was also enhanced. In addition, we know that the changes in the red-green fluorescence ratio of $\mathrm{JC}-1$ dye in cells can reflect alterations in mitochondrial membrane potential (17). It was observed that when miR-363-3p was overexpressed in the miR-363$3 \mathrm{p}$ mimic group, more cells fluoresced green than red after JC-1 staining, which was determined by flow cytometry. This also indicates that mitochondrial membrane potential was decreased in more cells, whereas the oxidative stress level in cells was increased. Therefore, these results further indicate that miR-363-3p enhances oxidative cellular oxidative stress. Moreover, cell cycle analysis showed that rat lung alveolar type II cells in the mimic group were arrested in the G1 phase. Therefore, based on the above flow cytometry analyses, we conclude that
miR-363-3p induces cell apoptosis by increasing cellular oxidative stress and G1-phase cell cycle arrest.

\section{Conclusions}

In summary, miR-363-3p is associated with lung cell proliferation and apoptosis. miR-363-3p inhibits rat lung alveolar type II cell proliferation by downregulating STRA6 expression and induces cell apoptosis by increasing cellular oxidative stress and G1-phase cell cycle arrest. However, this study was limited because the experiment was performed in vitro using immortalized rat lung alveolar type II cells. The next step will be to confirm the results of this study by evaluating entire lungs in vitro or conducting animal experiments. 

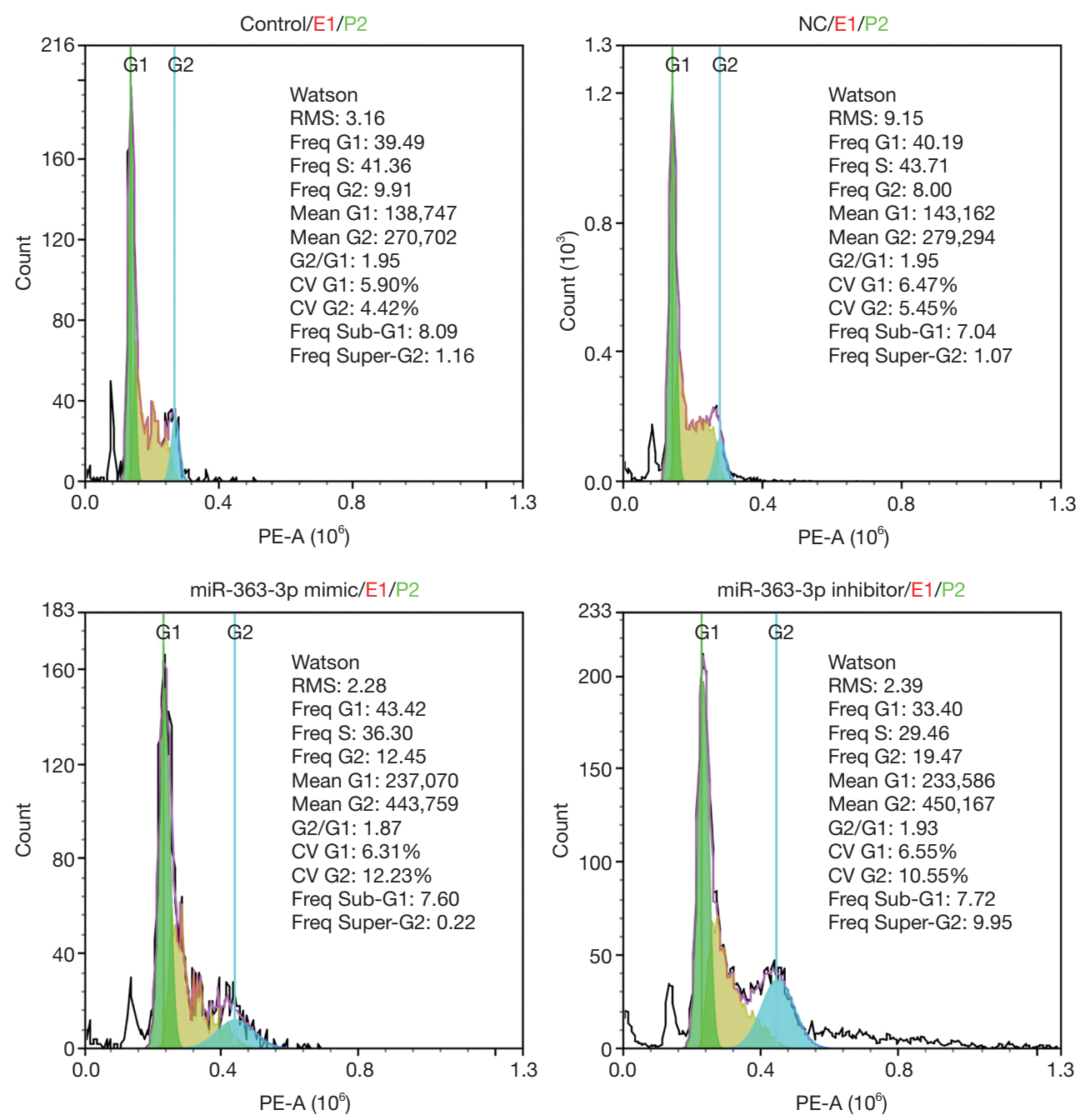

Figure 8 The Freq G1 value of the miR-363-3p mimic group was increased by 3.93 compared with that of the control group (43.42 "versus" 39.49), and the Freq G1 value of the miR-363-3p inhibitor group was decreased by 6.09 compared with that of the control group (39.49 "versus" 33.40).

\section{Acknowledgments}

Funding: This study was funded by the China Postdoctoral Science Foundation (Postdoc No. 241006) and Innovation Project of Women and Children Medical Research Center affiliated to Foshan Institute of Fetal Medicine (No. FEYJZX-2020-001).

\section{Footnote}

Reporting Checklist: The authors have completed the MDAR reporting checklist. Available at https://dx.doi. org/10.21037/tp-21-303
Data Sharing Statement: Available at https://dx.doi. org/10.21037/tp-21-303

Conflicts of Interest: All authors have completed the ICMJE uniform disclosure form (available at https://dx.doi. org/10.21037/tp-21-303). The authors have no conflicts of interest to declare.

Ethical Statement: The authors are accountable for all aspects of the work in ensuring that questions related to the accuracy or integrity of any part of the work are appropriately investigated and resolved. 
Open Access Statement: This is an Open Access article distributed in accordance with the Creative Commons Attribution-NonCommercial-NoDerivs 4.0 International License (CC BY-NC-ND 4.0), which permits the noncommercial replication and distribution of the article with the strict proviso that no changes or edits are made and the original work is properly cited (including links to both the formal publication through the relevant DOI and the license). See: https://creativecommons.org/licenses/by-nc-nd/4.0/.

\section{References}

1. Fernandes-Silva H, Vaz-Cunha P, Barbosa VB, et al. Retinoic acid regulates avian lung branching through a molecular network. Cell Mol Life Sci 2017;74:4599-619.

2. Coste K, Beurskens LW, Blanc P, et al. Metabolic disturbances of the vitamin A pathway in human diaphragmatic hernia. Am J Physiol Lung Cell Mol Physiol 2015;308:L147-57.

3. Kardon G, Ackerman KG, McCulley DJ, et al. Congenital diaphragmatic hernias: from genes to mechanisms to therapies. Dis Model Mech 2017;10:955-70.

4. Noy N. Vitamin A Transport and Cell Signaling by the Retinol-Binding Protein Receptor STRA6. Subcell Biochem 2016;81:77-93.

5. Zheng J, He Q, Tang H, et al. miR-455-5p Overexpression Reduces Rat Lung Alveolar Type II Cell Proliferation by Downregulating STRA6. Anat Rec (Hoboken) 2019;302:2062-9.

6. Zheng J, He Q, Tang H, et al. Overexpression of miR-455$5 \mathrm{p}$ affects retinol (vitamin A) absorption by downregulating STRA6 in a nitrofen-induced $\mathrm{CDH}$ with lung hypoplasia rat model. Pediatr Pulmonol 2020;55:1433-9.

7. Chen CH, Ke LY, Chan HC, et al. Electronegative low density lipoprotein induces renal apoptosis and fibrosis:

Cite this article as: Zheng J, Zhu S, Xu H, Li J, Tang H, Zhou Y, Huang Z, Liu G. miR-363-3p inhibits rat lung alveolar type II cell proliferation by downregulating STRA6 expression and induces cell apoptosis via cellular oxidative stress and G1-phase cell cycle arrest. Transl Pediatr 2021;10(8):2095-2105. doi: $10.21037 /$ tp-21-303
STRA6 signaling involved. J Lipid Res 2016;57:1435-46.

8. Ambros V. The functions of animal microRNAs. Nature 2004;431:350-5.

9. Lu Y, Thomson JM, Wong HY, et al. Transgenic overexpression of the microRNA miR-17-92 cluster promotes proliferation and inhibits differentiation of lung epithelial progenitor cells. Dev Biol. 2007;310:442-53.

10. Ventura A, Young AG, Winslow MM, et al. Targeted deletion reveals essential and overlapping functions of the miR-17 through 92 family of miRNA clusters. Cell. 2008; 132:875-86

11. Zhu S, He Q, Zhang R, et al. Decreased expression of miR-33 in fetal lungs of nitrofen-induced congenital diaphragmatic hernia rat model. J Pediatr Surg 2016;51:1096-100.

12. Bartel DP. MicroRNAs: genomics, biogenesis, mechanism, and function. Cell 2004;116:281-97.

13. Marquez HA, Chen F. Retinoic Acid Signaling and Development of the Respiratory System. Subcell Biochem 2020;95:151-74.

14. Kawaguchi R, Yu J, Honda J, et al. A membrane receptor for retinol binding protein mediates cellular uptake of vitamin A. Science 2007;315:820-5.

15. Pasutto F, Sticht H, Hammersen G, et al. Mutations in STRA6 cause a broad spectrum of malformations including anophthalmia, congenital heart defects, diaphragmatic hernia, alveolar capillary dysplasia, lung hypoplasia, and mental retardation. Am J Hum Genet 2007;80:550-60.

16. Green DR, Reed JC. Mitochondria and apoptosis. Science 1998;281:1309-12.

17. Salvioli S, Ardizzoni A, Franceschi C, et al. JC-1, but not DiOC6(3) or rhodamine 123, is a reliable fluorescent probe to assess delta psi changes in intact cells: implications for studies on mitochondrial functionality during apoptosis. FEBS Lett 1997;411:77-82. 